\title{
Uso de FAO-56 y cintilómetro para estimar la evapotranspiración de sorgo (Sorghum bicolor L.) y sus componentes: evaporación del suelo y transpiración de la planta Use of FAO-56 and scintillometer to estimate evapotranspiration of grain sorghum (Sorghum bicolor L.) and its components: soil evaporation and plant transpiration
}

\section{Jesús Enrique López Avendaño ${ }^{1, *}$, Julio César Rodríguez ${ }^{2}$, José Ángel Martínez Gallardo, Ramón Lizárraga Jiménez ${ }^{1}$ y Tomás Díaz Valdés ${ }^{1}$}

\footnotetext{
${ }^{1}$ Facultad de Agronomía, Universidad Autónoma de Sinaloa. Km 17.5 Carretera Culiacán-El Dorado. 80000 Culiacán, Sinaloa, México. Autor para correspondencia (profe.jesus@uas.edu.mx)

${ }^{2}$ División de Ciencias Biológicas y de la Salud, Departamento de Agricultura y Ganadería. Universidad de Sonora. Km 20.5 Carretera Hermosillo-Bahía Kino. 83000 Hermosillo, Sonora, México.
}

\section{RESUMEN}

Estimar la evapotranspiración de los cultivos (ETc) es esencial para la planificación y manejo del agua en una zona agrícola, particularmente en las regiones áridas y semiáridas; la separación de ETc en la evaporación del suelo (E) y la transpiración de la planta $(\mathrm{T})$ permitiría el uso de estrategias más eficientes de gestión del agua. Los objetivos del presente estudio fueron estimar la evapotranspiración diaria de sorgo (Sorghum bicolor L.) y sus componentes, utilizando los métodos FAO-56 con la aproximación del coeficiente dual de cultivo (Kc dual) y cintilómetro, así como, determinar los coeficientes de cultivo únicos (Kc) y basales (Kcb) para las diferentes etapas de desarrollo de sorgo. Los resultados demostraron que usar la aproximación del Kc dual permite una estimación razonable de ETc en comparación con los valores estimados utilizando el método del cintilómetro. La ETc estimada con ambos métodos fue 413.8 y $406.3 \mathrm{~mm}$, respectivamente, lo que arrojó una sobreestimación del 1.8\% para FAO-56. El error cuadrático medio (RMSE) entre ETc estimada fue $0.584 \mathrm{~mm} \mathrm{~d}^{-1} \mathrm{y}$ el coeficiente de concordancia de Willmott (d) fue 0.91. La evaporación representa el $17.1 \%$ de ETc estimada con FAO-56 y el $21.6 \%$ con el método del cintilómetro; el RMSE entre los valores de E estimados con ambos métodos fue $0.397 \mathrm{~mm} \mathrm{~d}^{-1} \mathrm{y}$ $\mathrm{d}=0.94$, mientras que, cuando se comparó $\mathrm{T}$, el RMSE calculado fue $0.371 \mathrm{~mm} \mathrm{~d}^{-1} \mathrm{y} \mathrm{d}=0.98$. Los valores de Kc estimados con el método FAO-56 fueron 0.68, 1.06 y 0.4 para las etapas inicial, media y final del cultivo, mientras que con el cintilómetro los valores fueron $0.75,0.94$ y 0.41 , respectivamente.

Palabras clave: balance de energía superficial, coeficiente dual de cultivo, scintilometría, Valle de Culiacán.

\section{SUMMARY}

Estimating evapotranspiration (ETc) is essential for water planning and management in agricultural areas, particularly in arid and semi-arid regions; ETc partitioning into soil evaporation (E) and plant transpiration $(\mathrm{T})$ would allow the use of more efficient water management strategies. The aims of the present study were to estimate daily evapotranspiration of sorghum and its components, using the FAO-56 method with the dual crop coefficient (dual $\mathrm{Kc}$ ) approach and scintillometer method, and to determine the single crop coefficient $(\mathrm{Kc})$ and basal crop coefficient $(\mathrm{Kcb})$ values for different development seasons. 
Results have shown that using the dual Kc approach allows reasonable simulation of ETc in comparison to values estimated using the scintillometer method. ETc estimated with both methods was 413.8 and $406.3 \mathrm{~mm}$, respectively, resulting in a $1.8 \%$ overestimation for FAO-56. Root mean square error (RMSE) was 0.584 $\mathrm{mm} \mathrm{d}^{-1}$ and Willmott's agreement coefficient (d) was 0.91. Evaporation estimated with FAO-56 was $17.1 \%$ of ETc, while with the scintillometer it was $21.6 \%$ of ETc. The RMSE of the estimated E values was 0.397 $\mathrm{mm} \mathrm{d}-1$ and $\mathrm{d}=0.94$, while, when comparing $\mathrm{T}$, calculated RMSE was $0.371 \mathrm{~mm} \mathrm{~d}-1$ and $\mathrm{d}=0.98 \mathrm{Kc}$ values estimated with the FAO-56 method were 0.68 , 1.06 and 0.4 for initial, middle and end season of the crop, while for the scintillometer, values were 0.75 , 0.94 and 0.41 , respectively.

Index words: surface energy balance, dual crop coefficient, scintillometry, Culiacan Valley.

\section{INTRODUCCIÓN}

En la actualidad, se requiere un entendimiento claro de la dependencia de los cultivos a la disponibilidad de agua y los posibles impactos de los sistemas agrícolas en la hidrología de una región (Yimam et al., 2015). El conocimiento de la evapotranspiración es esencial para los responsables de la planificación y manejo del agua en el sector agrícola, especialmente en las regiones áridas y semiáridas donde la demanda de agua de los cultivos supera la precipitación y se requiere la aplicación a través del riego. Esto es particularmente importante en áreas donde el uso del agua está regulado debido a programas de protección ecológica y existen recursos limitados que generan una alta competencia por el uso del vital líquido, lo que provoca un aumento en el grado de presión sobre los recursos hídricos. Es importante señalar que la evapotranspiración actual varía regional y temporalmente según las condiciones climáticas del área (López et al., 2015), y algunos autores señalan que estas condiciones son adversas para la producción agrícola (Marine et al., 2015).

La evapotranspiración consiste de dos procesos, la evaporación directa del suelo y la transpiración de la planta, y ambos ocurren simultáneamente, de modo que se han realizado importantes esfuerzos para cuantificarlos por separado (Er-Raki et al., 2010; Béziat et al., 2013; Zhang et al., 2013; Zhao et al., 2015). La transpiración de las plantas está directamente asociada a la producción de cultivos, mientras que la evaporación no contribuye a la producción en el campo, por lo que los dos procesos deben ser estudiados y cuantificados por separado para implementar estrategias de manejo del agua y programas de riego que permitan un uso eficiente (Ding et al., 2013).

Se ha desarrollado una amplia variedad de métodos y modelos para estimar con precisión la ETc, los cuales se han implementado a diversas escalas espaciales y temporales, desde el más simple (balance hídrico del suelo) hasta el más complejo (covarianza de vórtices, teledetección y scintilometría). Todos los métodos presentan limitaciones relacionadas con las condiciones del experimento, así como con la cantidad y calidad de los datos requeridos para la estimación (Er-Raki et al., 2013). El modelo más utilizado, por su simplicidad y robustez es el modelo FAO-56, que describe la relación entre la evapotranspiración del cultivo (ETc) y evapotranspiración de referencia (ETo) a través de un coeficiente de cultivo ( $\mathrm{Kc}$ ), que involucra los efectos de la transpiración de las plantas y la evaporación del suelo (Allen et al., 1998). Algunos investigadores han utilizado este modelo con el enfoque de Kc único (Martínez-Cob y Faci, 2010), mientras que otros han utilizado el modelo de FAO56 con el enfoque de Kc dual (Er-Raki et al., 2008). El último, separa el valor de Kc en un coeficiente de cultivo basal $(\mathrm{Kcb})$ dependiendo de las características fenotípicas, fisiológicas y morfológicas de la planta; y un coeficiente de evaporación del suelo (Ke), que básicamente, depende de la textura y densidad del suelo, así como del contenido de humedad en el mismo.

Con frecuencia, el sistema de covarianza de vórtices (CE) se utiliza en los estudios de evapotranspiración (ET) debido a su facilidad de configuración, la capacidad de proporcionar información continua $\mathrm{y}$ directa sobre los flujos turbulentos de superficie, y son sistemas automatizados (Zhang et al., 2013). Recientemente, algunos investigadores reportan el uso de CE para estimar ET (Gebler et al., 2015; Bolinius et al., 2016; Imukova et al., 2016), sin embargo, esta técnica es poco reportada para dividir la transpiración de la planta y la evaporación del suelo (Williams et al., 2004; Zhang et al., 2013). De manera similar, algunos estudios reportan el uso de la técnica de scintilometría para estimar la ET (Ezzahar et al., 2009; Cammalleri et al., 2010; Samain et al., 2012), pero ninguno para cuantificar la transpiración y la evaporación del suelo por separado. 
México ocupa el segundo lugar a nivel mundial en la producción de sorgo después de Estados Unidos. En el período 2012-2016, la producción promedio fue de 6.6 millones de toneladas, lo que representa el 10.7\% de la producción mundial; sin embargo, México también es un país importador de este cereal, toda vez que en el mismo período las importaciones promedio fueron de 652 mil toneladas (USDA, 2016). A nivel nacional, el sorgo se produce en condiciones de riego y secano; durante el período 2012-2016, el 35.5\% del área total de sorgo cosechada en México fue bajo condiciones de riego, produciendo el $47.3 \%$ de la producción total con un rendimiento promedio de $4.9 \mathrm{Mg} \mathrm{ha}^{-1}$, mientras que $64.5 \%$ de la superficie total cosechada de sorgo se sembró bajo condiciones de temporal (secano), lo que produjo un $52.7 \%$ de la producción total con un rendimiento promedio de $3 \mathrm{Mg} \mathrm{ha}^{-1}$. El cultivo de sorgo grano ocupa el tercer lugar de la superficie cultivada en México, después del maíz y el trigo. Durante el período 2012-2016, la superficie promedio cosechada de este cereal, a nivel nacional, fue 324612 ha, que representa el $15.2 \%$ de la superficie agrícola total cosechada, Al respecto, Sinaloa es el segundo mayor productor de sorgo en México, y aporta el $25.8 \%$ de la superficie cosechada a nivel nacional, contribuyendo con el $30 \%$ de la producción total de este cultivo (SIAP, 2016). En el Valle de Culiacán, donde se localiza el Distrito de Riego 010 "Culiacán-Humaya", la superficie promedio anual sembrada con sorgo grano para el periodo 2012-2016 fue 10963 ha, con un volumen neto promedio de agua aplicada de $74423 \mathrm{hm}^{3}$, lo cual representa una lámina anual de riego de $688 \mathrm{~mm}$ (SEMARNAT-CONAGUA, 2017).

Para el presente trabajo se definieron dos objetivos principales: el primero, consistió en estimar la evapotranspiración del sorgo grano y sus componentes: evaporación del suelo y transpiración de la planta, utilizando el método FAO-56 con Kc dual y el método del cintilómetro; el segundo fue determinar los coeficientes de cultivos únicos y basales para el sorgo grano.

\section{MATERIALES Y MÉTODOS}

\section{Descripción del Sitio}

El trabajo de campo se realizó en la empresa agrícola "El Chaparral", con 80 ha sembradas de sorgo grano, localizadas en el Valle de Culiacán, al noroeste de México con coordenadas geográficas de $24.6 \mathrm{~N}$ y $107.588^{\circ} \mathrm{O}$ (Figura 1). La zona es semiárida con una precipitación media anual total de $690 \mathrm{~mm}$; la temperatura promedio anual es de $25{ }^{\circ} \mathrm{C}$, llegando a $43{ }^{\circ} \mathrm{C}$ en el verano (julio) y bajando a $0.6^{\circ} \mathrm{C}$ en invierno (enero). El suelo predominante es textura francoarcillo-limosa, con densidad aparente de $1.48 \mathrm{~g} \mathrm{~cm}^{-3}$. El período considerado para la estimación fue de 120 días a partir del 20 de marzo (Día del año, DOY 79) hasta el 17 de julio (DOY 198) del año 2011.

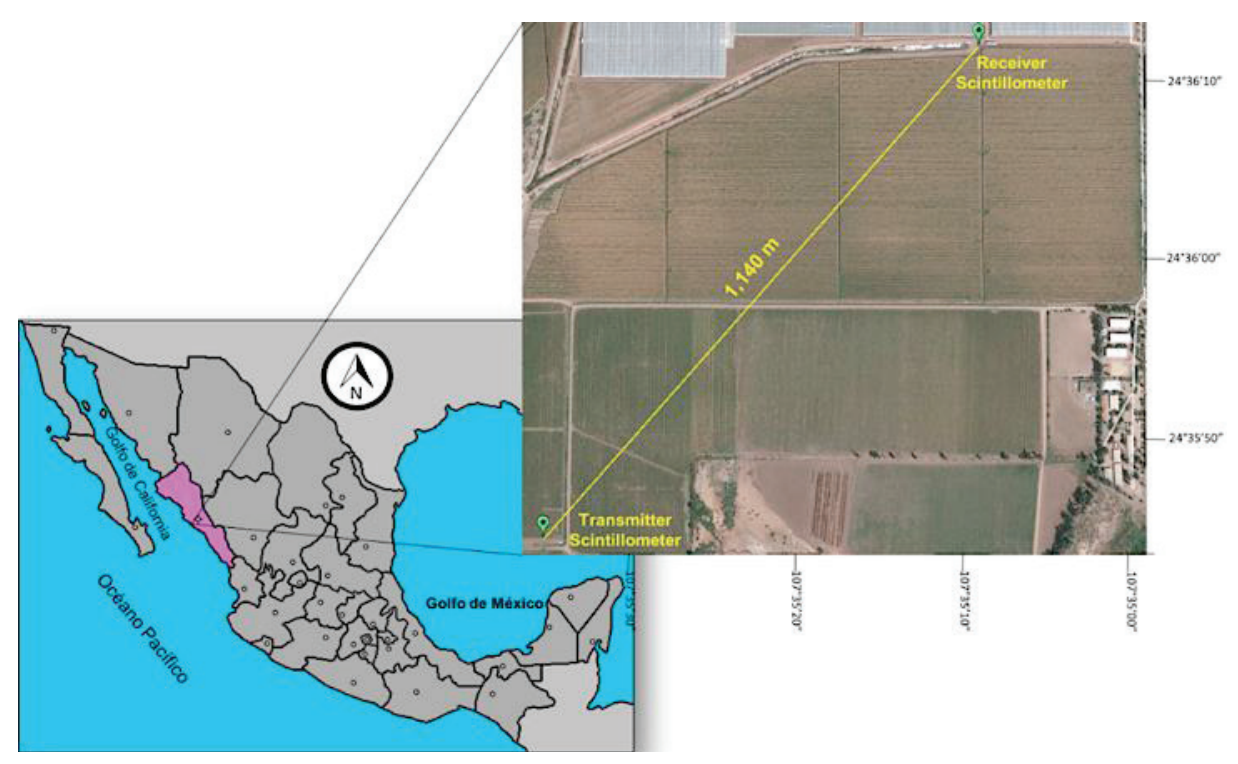

Figura 1. Localización del sitio de estudio y posición del cintilómetro. Figure 1. Location of the study site and position of the scintillometer. 


\section{Datos Meteorológicos y Medición con el Cintilómetro}

Se instaló una estación meteorológica en el sitio de estudio, donde se colocaron sensores para medir las variables meteorológicas necesarias para la estimación de la ETc. Los datos meteorológicos se consideraron por periodos de $10 \mathrm{~min}$ y fueron la radiación neta, temperatura del aire, humedad relativa, velocidad y dirección del viento, presión barométrica, lluvia y flujo de calor del suelo. Los ajustes de datos se realizaron de acuerdo con el procedimiento descrito por Allen et al. (1998); por otro lado, se utilizó un cintilómetro BLS450 (Scintec ${ }^{\circledR)}$ para estimar el flujo de calor sensible en la superficie $(\mathrm{H})$.

\section{Estimación de ETc con el Método de la FAO Penman- Monteith (FAO-56) Utilizando la Aproximación de Kc Dual y la Separación de la Evaporación del Suelo (E) y Transpiración de la Planta (T)}

La evapotranspiración real del cultivo (ETc) se calculó con la siguiente expresión:

$$
E T_{C}=E T_{0}(K s K c b+K e)
$$

donde: ETc es la evapotranspiración real del cultivo $\left(\mathrm{mm} \mathrm{d}^{-1}\right)$, ETo es la evapotranspiración de referencia $\left(\mathrm{mm} \mathrm{d}^{-1}\right)$; Kcb es el coeficiente basal de cultivo que incluye el concepto de transpiración del cultivo, Ks es un coeficiente de estrés hídrico y Ke es el coeficiente de evaporación superficial. La ETo se calculó con la ecuación de la FAO Penman-Monteith ajustada por períodos de 10 min (Ecuación 2) e integrada día a día.

$E T \mathrm{O}=\frac{0.408 \Delta\left(R_{n}-G\right)+\gamma\left(\frac{6.25}{T+273}\right) u_{2}\left(e_{s}-e_{a}\right)}{\Delta+\gamma\left(1+0.34 u_{2}\right)}$

donde: $\mathrm{Rn}$ es la radiación neta $\left(\mathrm{MJ} \mathrm{m}^{-2}\right), \mathrm{G}$ es el flujo de calor del suelo ( $\left.\mathrm{MJ} \mathrm{m}^{-2}\right), \Delta$ es la pendiente de la curva de presión de vapor $\left(\mathrm{KPa}{ }^{\circ} \mathrm{C}^{-1}\right), \gamma$ es la constante psicrométrica $\left(\mathrm{kPa}^{\circ} \mathrm{C}^{-1}\right), \mathrm{u}_{2}$ es la velocidad del viento a $2 \mathrm{~m}$ de altura $\left(\mathrm{m} \mathrm{s}^{-1}\right),\left(\mathrm{e}_{\mathrm{s}}-\mathrm{e}_{\mathrm{a}}\right)$ es el déficit de presión de vapor $(\mathrm{kPa})$ y $\mathrm{T}$ es la temperatura del aire $\left({ }^{\circ} \mathrm{C}\right)$.

Para el método FAO-56, se utilizaron las ecuaciones propuestas por Allen et al. (1998), con las cuales se calcularon y ajustaron los valores de los coeficientes requeridos, incluyendo el coeficiente basal de cultivo $(\mathrm{Kcb})$, coeficiente de cultivo máximo $\left(\mathrm{Kc}_{\max }\right)$, coeficiente de evaporación del suelo $(\mathrm{Ke})$, coeficiente de reducción de la evaporación $(\mathrm{Kr})$, coeficiente de estrés hídrico (Ks), humedad totalmente evaporable (TEW), humedad fácilmente evaporable (REW), humedad totalmente disponible en la zona radicular (TAW) y la humedad fácilmente disponible en la zona radicular (RAW).

Los valores para humedad a capacidad de campo $\left(\mathrm{q}_{\mathrm{cc}}\right)$, humedad a punto de marchitez permanente $\left(\mathrm{q}_{\mathrm{pmp}}\right)$ y capa del suelo sujeta a la evaporación (Ze) en el sitio de estudio fueron $0.405 \mathrm{~cm}^{3} \mathrm{~cm}^{-3}, 0.242 \mathrm{~cm}^{3} \mathrm{~cm}^{-3} \mathrm{y}$ $0.15 \mathrm{~m}$, respectivamente. Para TEW y REW, los valores calculados fueron 42.6 y $19.2 \mathrm{~mm}$, respectivamente, los cuales están de acuerdo con los valores típicos de Allen et al. (1998); la fracción de suelo expuesta y húmeda $\left(\mathrm{f}_{\mathrm{ew}}\right)$ varió de 0.97 en la etapa inicial, donde la cobertura de planta fue mínima, hasta casi cero cuando el cultivo cubrió completamente el suelo (0.05). Los valores de TAW y RAW calculados fueron 244.5 y $134.5 \mathrm{~mm}$, respectivamente; la profundidad efectiva de raíz para el sorgo se fijó en $1.5 \mathrm{~m}$ y para la fracción de agotamiento hídrico (p) fue de 0.55 (Allen et al., 1998).

\section{Estimación de ETc con Cintilómetro (BLS) y Separación de la Evaporación del Suelo (E) y Transpiración de la Planta (T)}

La estimación de ET utilizando la ecuación de balance de energía de la superficie del suelo se realizó usando un cintilómetro BLS450. E1 BLS es un dispositivo utilizado para derivar la intensidad de la turbulencia superficial a través de la medición del índice de refracción del aire $\left(\mathrm{Cn}^{2}\right)$. La teoría de cintilometría que incluye la teoría MOST (Teoría de Similaridad de Monin-Obukhov) y las ecuaciones utilizadas para este experimento pueden revisarse en Liu et al. (2013). La ecuación final para calcular $\mathrm{H}$ se escribe como:

$H=\rho_{a} C_{p} u \cdot T$.

donde: $\mathrm{H}$ es el flujo de calor sensible $\left(\mathrm{w} \mathrm{m}^{-2}\right)$, $\rho$ a es la densidad del aire $\left(\mathrm{kg} \mathrm{m}^{-3}\right), \mathrm{C}_{\mathrm{p}}$ es la capacidad de calor especifico del aire a presión constante $\left(\mathrm{J} \mathrm{kg}^{-1}{ }^{\circ} \mathrm{K}^{-1}\right), \mathrm{u}_{*}$ es la velocidad de fricción $\left(\mathrm{m} \mathrm{s}^{-1}\right)$ y $\mathrm{T}_{*}$ es un escalar de la temperatura $\left({ }^{\circ} \mathrm{K}\right)$.

Se verificó la información obtenida con el instrumento para garantizar la calidad de los datos del BLS, para ello se consideró lo siguiente: se rechazaron 
los datos de $\mathrm{Cn}^{2}$ bajo condiciones de saturación atmosférica obtenida durante la noche, considerando solamente los datos de las 7:00 a las 17:00 h por día; además, se rechazaron los datos que se recopilaron cuando el sensor no funcionaba correctamente. Las mediciones se realizaron cada minuto y se obtuvo el promedio por cada 10 minutos para el procesamiento final.

Una vez estimado el flujo de calor sensible $(\mathrm{H})$ se calculó el flujo de calor latente (LE, $\mathrm{w} \mathrm{m}^{-2}$ ) a partir de la ecuación del balance de energía mediante la medición de la radiación neta $\left(\mathrm{Rn}, \mathrm{w} \mathrm{m}^{-2}\right)$ y flujo de calor de la superficie del suelo $\left(\mathrm{G}, \mathrm{w} \mathrm{m}^{-2}\right)$ con la expresión:

$$
E=R_{n}-G-H
$$

Se utilizó el calor latente de vaporización (2,45 MJ) para convertir LE a un valor equivalente de lámina de agua (Allen et al., 1998). Después de estimar ETc con el método del cintilómetro, se calcularon los coeficientes $\mathrm{Ke}$, Ks y Kcb. Al considerar que Ks depende de las condiciones de riego del cultivo y de la capacidad de retención de agua del suelo, se tomaron en cuenta los mismos valores de Ks calculados con FAO-56.

Para el cálculo de Ke, la relación entre ETc estimada con BLS (ETc $\left.{ }_{\text {BLS }}\right)$, ETo, Ks y Kcb ajustado se utilizó de la siguiente manera:

$K_{e}=\frac{E T_{c B L S}}{E T_{0}}-K_{s} K_{c b a d j}$

Una vez calculado Ke, la evaporación del suelo (E) se estimó con la siguiente expresión:

$E=E T_{0} K e$

A su vez, la transpiración de la planta (T) se calculó mediante la ecuación siguiente:

$T=E T_{c B L S}-E$

Finalmente, el coeficiente basal del cultivo calculado con ETc estimada con BLS $\left(\mathrm{Kcb}_{\mathrm{BLS}}\right)$ se calculó con la expresión:

$K_{c b B L S}=\frac{T}{E T_{0} K_{s}}$

\section{Evaluación Estadística de los Modelos}

El comportamiento de los modelos se evaluó utilizando varios indicadores de ajuste, siguiendo el procedimiento realizado en otros estudios previos sobre balance de agua y energía. i) Raíz media del error cuadrático (RMSE) caracteriza la varianza de los errores. ii) El error absoluto promedio (EAP) expresa la magnitud de los errores de estimación en alternativa al RMSE. iii) El índice de concordancia de Willmott (d) representa la relación entre el error cuadrático medio y el "error potencial". iv) BIAS indica la desviación promedio de los valores estimados de aquellos considerados como valores observados o medidos. v) La eficiencia de modelación de Nash y Sutcliffe (Ef) define la relación entre el error cuadrático medio y la varianza en los datos observados restados de la unidad. Vi) Coeficiente de correlación (R) (Er-Raki et al., 2010; Zhang et al., 2013; Zhao et al., 2015).

\section{RESULTADOS Y DISCUSIÓN}

\section{Estimación de la Evapotranspiración Real (ETc)}

La variación temporal de ETc se estimó utilizando el método FAO-56 con la aproximación del coeficiente dual de cultivo (Kc dual), y el método del cintilómetro usando un BLS450, durante todo el ciclo vegetativo de sorgo grano de primavera-verano (P-V) en el Valle de Culiacán, México. La Figura 2 presenta la variación temporal de ETc estimada con ambos métodos; para la ETc estimada con FAO-56, se utilizó la aproximación con Kc dual y los valores calculados diariamente de Ke y Kcb, donde se muestran claramente las diferencias en la etapa inicial del cultivo, durante esta etapa, el método FAO56 refleja un valor alto de ETc diaria, afectada por la evaporación directa del suelo; sin embargo, la humedad disponible en la capa de evaporación disminuye con el tiempo y ETc se reduce drásticamente, debido al bajo factor de cobertura vegetal (fc).

El comportamiento de ETc estimada con BLS se ajusta mejor a ETo. La ETc diaria estimada con BLS no refleja la misma tendencia de cambio que la ETc diaria estimada con FAO-56, lo cual posiblemente se debe a la gran cantidad de datos de suelo y cultivo requeridos, y a la escala local de este método. Cabe hacer notar 


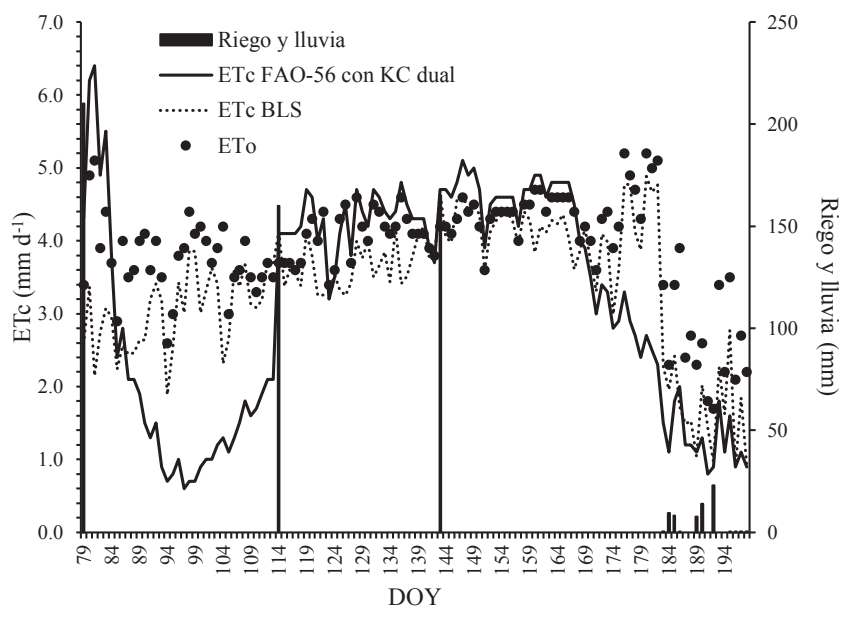

Figura 2. Evolución de la ET $_{0}$ y ETc diaria estimada con FAO-56 y BLS. Para el método FAO-56 se utilizó el Kcb estimado diariamente durante el desarrollo del cultivo.

Figure 2. Evolution of ET and ETc estimated daily with FAO-56 and BLS. For the FAO-56 method, the estimated Kcb was used daily during crop development.

que el uso del cintilómetro y su técnica de cálculo es una medida con mayor cobertura (escala regional) que se basa únicamente en el balance de energía y que este método no tiene una capa de evaporación como límite al proceso. En la Figura 3 se observa un mejor ajuste de los resultados obtenidos con ambos métodos, al utilizar los valores de Kcb y Ke promedio por etapa

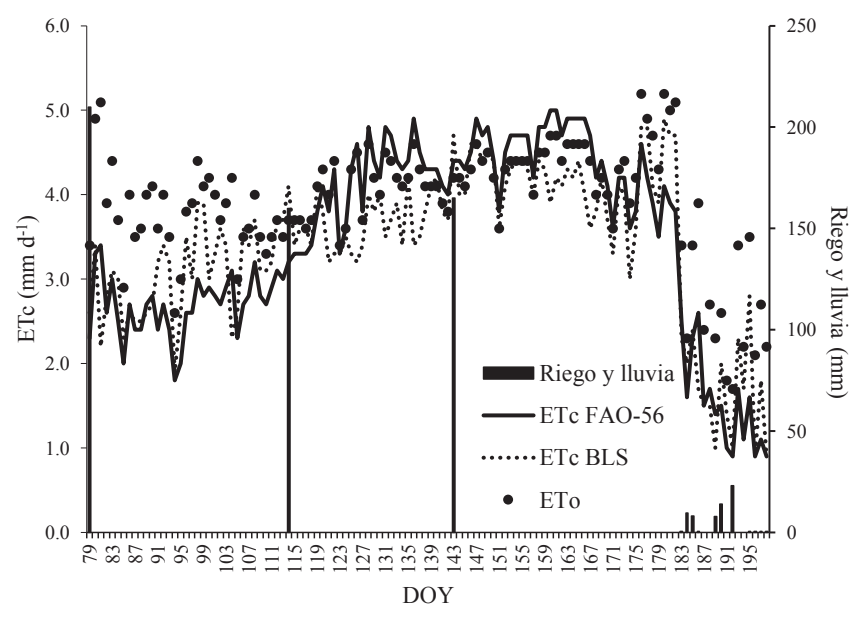

Figura 3. Evolución de ET $_{0}$ y la ETc diaria estimada con FAO-56 y BLS. Para el método FAO-56 se utilizó el Kcb promedio por etapa de desarrollo del cultivo.

Figure 3. Evolution of ET $_{0}$ and the daily ETc estimated with FAO-56 and BLS. For the FAO-56 method, the average Kcb was used per crop development stage. de desarrollo del cultivo para el método FAO56 (Kcb de $0.18,1.05$ y 0.40 , para las etapas inicial, media y final, respectivamente, mientras que para los valores de Ke fueron $0.50,0.01$ y 0.03 para las mismas etapas de desarrollo).

La ETc total estimada con FAO-56 utilizando Kc dual promedio por etapa de desarrollo y cintilómetro fue 413.8 y $406.3 \mathrm{~mm}$, respectivamente, lo que representa una sobreestimación del 1.8\% del método FAO-56. Estos valores de ETc en sorgo grano son más bajos que otros obtenidos en diferentes sitios del mundo: Bashir et al. (2008) obtuvieron 579 y $674 \mathrm{~mm}$ en Sudán, África. Piccinni et al. (2009) estimaron 491 y $533 \mathrm{~mm}$ en Texas, EE. UU; Sánchez et al. (2011) calcularon 500 y $524 \mathrm{~mm}$ en España; Shenkut et al. (2013) obtuvieron $500.7 \mathrm{~mm}$ en Etiopía y López et al. (2016) obtuvieron 721 y $691 \mathrm{~mm}$ en el área de Albacete, España. Es posible inferir que las diferencias en los resultados obtenidos en esta investigación con respecto a los estudios mencionados se deben a la duración del ciclo vegetativo considerado y a las diversas condiciones climatológicas de los sitios de estudio que aumentan la demanda evaporativa de la atmósfera, además de las diferencias por los propios métodos de estimación utilizados. El riego total aplicado y la lámina de lluvia registrada fueron de $535 \mathrm{~mm}$ y $65.2 \mathrm{~mm}$, respectivamente. La eficiencia del riego por surcos fue de 77 y $76 \%$ aplicando los valores de ETc estimados con FAO-56 y BLS, respectivamente.

La comparación entre ETc calculada utilizando el método FAO-56 y la aproximación de Kc dual presenta un buen ajuste con la ETc estimada usando BLS (Figura 3). La raíz cuadrada del error cuadrático (RMSE) entre los dos valores fue $0.584 \mathrm{~mm} \mathrm{~d}^{-1}$, el coeficiente de concordancia de Willmott (d) fue de 0.91, la eficiencia de Nash y Sutcliffe (Ef) fue 0.59, EAP fue $0.475 \mathrm{~mm} \mathrm{~d}^{-1}$, BIAS fue $0.052 \mathrm{~mm} \mathrm{~d}^{-1} \mathrm{y}$ el coeficiente de correlación $(\mathrm{R})$ fue 0.86 . Dichos resultados se ajustan de mejor manera que los reportados en Sánchez et al. (2011), quienes compararon los valores de ETc para el sorgo obtenido con el método TSEB (TwoSource Energy Balance) y lisímetro, donde RMSE $=1.03 \mathrm{~mm} \mathrm{~d}^{-1}$, BIAS $=0.3 \mathrm{~mm} \mathrm{~d}^{-1} \mathrm{y} \mathrm{R}=0.89$. En otra investigación, Er-Raki et al. (2010) compararon el método FAO-56 con la técnica de covarianza de vórtices para la estimación de ETc en olivo, y encontraron valores de RMSE de 0.54 y $0.71 \mathrm{~mm} \mathrm{~d}^{-1}, \mathrm{Ef}=0.58$ y 0.59 y BIAS $=0.02$ y $0.05 \mathrm{~mm} \mathrm{~d}^{-1}$. Por su parte, Zhang et al. (2013) estimaron ETc para trigo y maíz, 
obteniendo valores de RMSE $=0.55 \mathrm{~mm} \mathrm{~d}^{-1}, \mathrm{~d}=0.97$ y $\mathrm{EAP}=0.42 \mathrm{~mm} \mathrm{~d}^{-1}$ y RMSE $=0.52 \mathrm{~mm} \mathrm{~d}^{-1}, \mathrm{~d}=0.95$, $\mathrm{EAP}=0.40 \mathrm{~mm} \mathrm{~d}^{-1}$, para cada cultivo respectivamente, utilizando los mismos dos métodos. Los resultados estadísticos obtenidos en la presente investigación son similares a los obtenidos por los autores citados anteriormente al comparar el método FAO-56 con el método directo de covarianza de vórtices.

\section{Determinación del Coeficiente Dual de Cultivo (Kc dual) y Coeficiente Único de Cultivo (Kc)}

Para dividir la ETc en evaporación del suelo y transpiración de la planta, se requirió determinar los coeficientes de evaporación del suelo $(\mathrm{Ke})$ y coeficiente basal del cultivo $(\mathrm{Kcb})$. Para el método FAO-56 se siguió la metodología propuesta por Allen et al. (1998) y para el cintilómetro se utilizaron las ecuaciones (5) y (8). En la Cuadro 1 se muestran los resultados estimados con ambos métodos y los valores tabulados propuestos por Allen et al. (1998) para las etapas inicial (ini), media (med) y final (fin). Asimismo, se determinaron los coeficientes únicos de cultivo (Kc) con ambos métodos, para ello se utilizó la relación $\mathrm{Kc}=\mathrm{ETc} / \mathrm{ET}_{0}$. Los resultados se muestran en la Cuadro 1.

Los valores de Kcb y Kc obtenidos en esta investigación para las etapas de desarrollo inicial, media y final son diferentes a los propuestos por Allen et al. (1998). Otros investigadores estimaron diferentes valores para las mismas etapas de desarrollo de sorgo: Bashir et al. (2008) indicaron valores de Kc de 0.62, 1.15 y 0.48 estimados mediante percepción remota, mientras que con el lisímetro obtuvieron valores de $\mathrm{Kc}$ de $0.55,1.21$ y 0.65 . Piccinni et al. (2009) calcularon valores para $\mathrm{Kc}$ de $0.40,0.80$ y 0.75 , mientras que, Shenkut et al. (2013) estimaron valores de 0.45, 1.18 y 0.78 Kc. López et al. (2016) determinaron valores de Kc de 0.64 y 1.19 para las etapas de desarrollo inicial y media, respectivamente, mientras que los valores de $\mathrm{Kcb}$ para esas mismas etapas fueron de 0.11 y 1.17 . Las diferencias que muestran los valores de Kc se deben a las condiciones de manejo del cultivo, las características de la variedad de cultivo y las condiciones climáticas locales (Pereira et al., 2015).

\section{Separación de ETc en Evaporación del Suelo (E) y Transpiración de la Planta (T)}

Para la separación de E y T de la ETc calculada con el método del cintilómetro (BLS), se consideró que el método FAO-56 con Kc dual estima con una precisión aceptable los valores de ETc total y la transpiración de la planta (T) (Liu y Luo, 2010), aunque cabe aclarar que se han observado algunas diferencias entre E calculada con FAO-56 y otros métodos evaluados, incluyendo el método de covarianza de vórtices y lisímetros (Er-Raki et al., 2010; Zhang et al., 2013).

E fue el principal componente de la ETc durante la etapa inicial de cultivo (Figura 4), con una relación E/ETc promedio de 74 para FAO56 y $76.3 \%$ para BLS (Cuadro 2). El valor alto de E en la etapa inicial del cultivo se debe a la baja transpiración (baja cobertura vegetal) y debido a la presencia de suficiente humedad en la capa superficial para satisfacer la demanda evaporativa de la atmósfera. Conforme la cobertura vegetal se incrementa durante las etapas de desarrollo, la relación E/ETc se redujo a 28.9 y $34.1 \%$ para FAO56 y BLS, respectivamente. Mientras que, durante la etapa media, $\mathrm{T}$ y fc se incrementaron a sus valores máximos (Figura 4), y la relación E/ETc se redujo al valor mínimo, 0.9 y $0.2 \%$, para ambos métodos evaluados, respectivamente. En la etapa final, con la senescencia de hojas, aunque el valor de T disminuyó,

Cuadro 1. Valores de Kcb y Kc estimados para sorgo grano (P-V) en el Valle de Culiacán, México. Table 1. Values of Kcb and Kc estimated for grain sorghum (P-V) in the Culiacán Valley, Mexico.

\begin{tabular}{lccccccc}
\hline \multirow{2}{*}{$\begin{array}{l}\text { Etapa de } \\
\text { desarrollo }\end{array}$} & \multicolumn{3}{c}{ Kcb } & & \multicolumn{3}{c}{ Kc } \\
\cline { 2 - 3 } \cline { 6 - 7 } & $\begin{array}{c}\text { Allen } \text { et al. } \\
(1998)\end{array}$ & FAO-56 & BLS & & $\begin{array}{c}\text { Allen } \text { et al. } \\
(1998)\end{array}$ & FAO-56 & BLS \\
\hline Inicial & 0.15 & 0.18 & 0.18 & & 0.7 & 0.68 & 0.75 \\
Medio & 1.1 & 1.06 & 0.95 & & 1.1 & 1.06 & 0.94 \\
Fin & 0.35 & 0.39 & 0.39 & & 0.55 & 0.4 & 0.41 \\
\hline
\end{tabular}

$\mathrm{Kcb}=$ coeficiente de cultivo basal; $\mathrm{Kc}=$ coeficiente de cultivo. $\mathrm{Kcb}=$ basal crop coefficient; $\mathrm{Kc}=$ cultivation coefficient. 

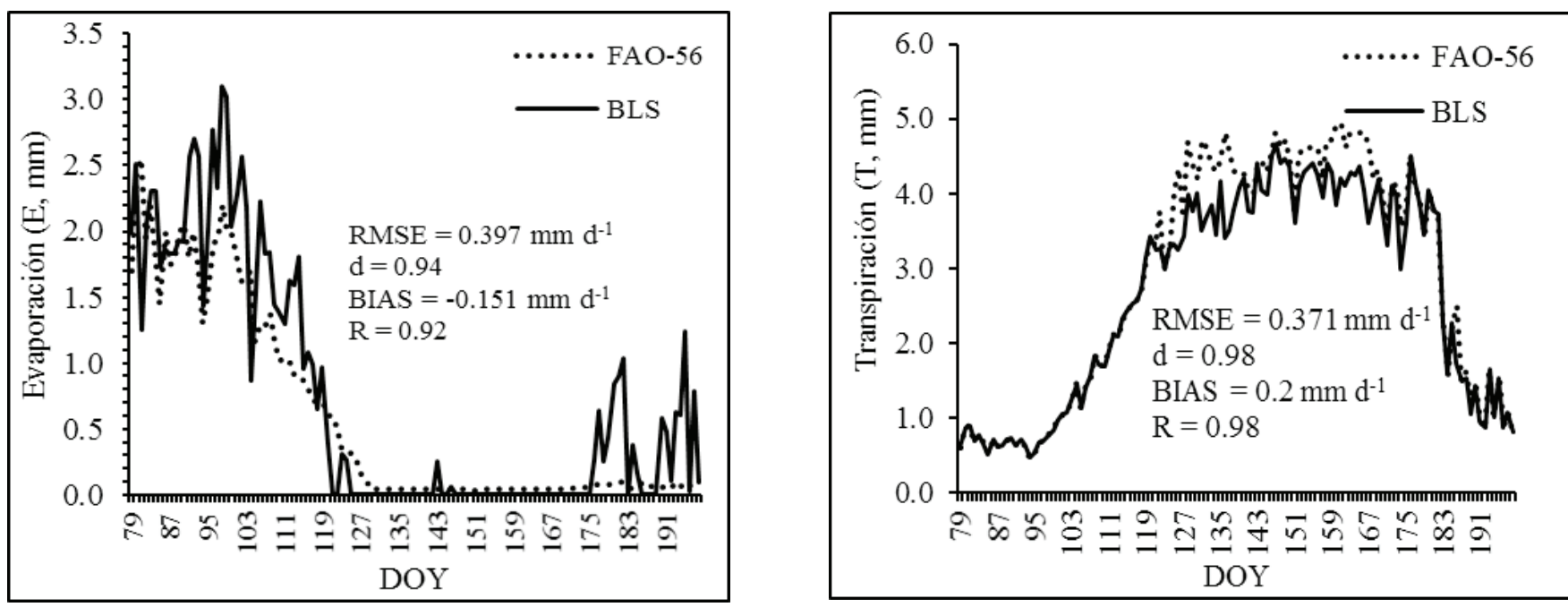

Figura 4. Comportamiento temporal de la evaporación del suelo (E) y transpiración de la planta (T), estimadas con los métodos FAO-56 y BLS.

Figure 4. Temporal behavior of soil evaporation (E) and transpiration of the plant (T) estimated with FAO-56 and BLS methods.

fc fue alto, por lo que la relación E/ETc se mantuvo baja (2.5 y $11.2 \%$, para FAO-56 y BLS, respectivamente), probablemente esto se debe a la presencia de lluvia durante la etapa final de cultivo.

Los resultados obtenidos con la presente investigación indican que la relación E/ETc total fue $17.1 \%$ para FAO56 utilizando Kc dual y $21.6 \%$ para BLS.

López et al. (2016) en la zona de Albacete (centro de España) determinaron con la medición mediante lisímetro, que la evaporación en sorgo representa el $18 \%$ de la ETc total medida, este valor concuerda con los resultados de esta investigación. En otros estudios con trigo y maíz, se reportaron otros valores de la relación E/ETc total; por ejemplo, Zhang et al. (2013) reportaron $28 \%$ para el trigo de invierno y $35-45 \%$ para el maíz de verano en China; Rosa et al. (2012) obtuvieron $25 \%$ para el trigo y el $16 \%$ para el maíz en Portugal; mientras que, Sadras y Rodríguez (2010) determinaron 22-58\% para el trigo en Australia.

Cuadro 2. Evaporación (E), transpiración (T) y evapotranspiración de real (ETc) por etapa de desarrollo del cultivo de sorgo grano en el Valle de Culiacán, México.

Table 2. Evaporation (E), transpiration (T) and evapotranspiration of real (ETc) by stage of development of the sorghum grain crop in the Culiacán Valley, Mexico.

\begin{tabular}{lcccccc}
\hline & & Inicial & Desarrollo & Media & Final & Total \\
\hline FAO-56 & E (mm) & 38.2 & 28.9 & 1.7 & 2.0 & 70.8 \\
& T (mm) & 13.4 & 71.1 & 180.4 & 78.2 & 343.0 \\
& ETc (mm) & 51.5 & 100.0 & 182.1 & 80.2 & 413.8 \\
BLS & E/ETo (\%) & 74.0 & 28.9 & 0.9 & 2.5 & 17.1 \\
& E (mm) & 43.0 & 35.1 & 0.3 & 9.5 & 87.9 \\
& T (mm) & 13.0 & 67.8 & 161.8 & 75.4 & 318.4 \\
& ETc (mm) & 56.4 & 102.9 & 162.1 & 84.9 & 406.3 \\
& E/ETo (\%) & 76.3 & 34.1 & 0.2 & 11.2 & 21.6 \\
\hline
\end{tabular}




\section{CONCLUSIONES}

- La ETc total estimada para sorgo grano en primaveraverano con un ciclo vegetativo de 120 días fue 413.8 y $406.3 \mathrm{~mm}$ para los métodos FAO-56 y cintilómetro, respectivamente, donde el error relativo (ER) entre ambos resultados fue $1.8 \%$, sobrestimándose ligeramente el valor con el método FAO-56. El desglose de los componentes fue para la evaporación del suelo (E) 70.8 y $87.9 \mathrm{~mm}$, lo que representa 17.1 y $21.6 \%$ de ETc total, para los métodos FAO56 y cintilómetro, respectivamente. Por su parte, la transpiración de la planta $(\mathrm{T})$ se estimó en $343.0 \mathrm{~mm}$ para FAO-56 y $318.4 \mathrm{~mm}$ para cintilómetro.

- Los valores calculados de Kc único para las etapas inicial, media y final del cultivo fueron $0.75,0.94$ y 0.41 ; mientras que los valores para $\mathrm{Kcb}$ fueron $0.18,0.95$ y 0.39 , respectivamente. Sin embargo, en términos prácticos se recomienda que para sorgo grano (P-V) en el Valle de Culiacán, los valores de Kc único definitivo para la etapa inicial, media y final es $0.75,0.95$ y 0.40 , respectivamente, mientras que los valores de Kcb son $0.20,0.95$ y 0.40 para las mismas etapas de desarrollo.

\section{AGRADECIMIENTOS}

Se agradece a la empresa agrícola "El Chaparral" por las facilidades otorgadas para la realización del trabajo de campo en este proyecto y al Organismo de Cuenca Pacífico Norte de la Comisión Nacional del Agua por las facilidades para el uso de instrumentos de medición (cintilómetro).

\section{LITERATURA CITADA}

Allen, R. G., L. S. Pereira, D. Raes, and M. Smith. 1998. Crop evapotranspiration - Guidelines for computing water requirements - FAO Irrigation and drainage paper 56. FAO. Rome. ISBN 92-5-104219-5.

Bashir, M. A., T. Hata, H. Tanakamaru, A. W. Abdelhadi, and A. Tada. 2008. Satellite-based energy balance model to estimate seasonal evapotranspiration for irrigated sorghum: A case study from the Gezira scheme, Sudan. Hydrol. Earth Syst. Sci 12: 1129-1139. doi: 10.5194/hess-12-1129-2008.

Béziat, P., V. Rivalland, T. Tallec, N. Jarosz, G. Boulet, P. Gentine, and E. Ceschia. 2013. Evaluation of a sample approach for crop evapotranspiration partitioning and analysis of the water budget distribution for several crop species. Agric. For. Meteorol. 177: 46-56. doi: 10.1016/j.agrformet.2013.03.013.
Bolinius, D. J., A. Jahnke, and M. MacLeod. 2016. Comparison of eddy covariance and modified Bowen ratio methods for measuring gas fluxes and implications for measuring fluxes of persistent organic pollutants. Atmos. Chem. Phys. 16: 5315-5322.

Cammalleri, C., C. Agnese, G. Ciraolo, M. Minacapilli, G. Provenzano, and G. Rallo. 2010. Actual evapotranspiration assessment by means of a coupled energy/hydrologic balance model: Validation over an olive grove by means of scintillometry and measurement of soil water contents. J. Hydrol. 392: 70-82.

Ding, R., S. Kang, Y. Zhang, X. Hao, L. Tong, and T. Du. 2013. Partitioning evapotranspiration into soil evaporation and transpiration using a modified dual crop coefficient model in irrigated maize field with ground-mulching. Agric. Water Manage. 127: 85-96. doi: 10.1016/j.agwat.2013.05.018.

Er-Raki, S., A. Chehbouni, J. Hoedjes, J. Ezzahar, B. Duchemin, and F. Jacob. 2008. Improvement of FAO-56 method for olive orchards through sequential assimilation of thermal infraredbased estimates of ET. Agric. Water Manage. 95: 309-321.

Er-Raki, S., A. Chehbouni, G. Boulet, and D. G. Williams. 2010. Using the dual approach of FAO-56 for partitioning ET into soil and plant components for olive orchards in semi-arid region. Agric. Water Manage. 97: 1769-1778.

Er-Raki, S., J. C. Rodríguez, J. Garatuza-Payán, C. J. Watts, and A. Chehbouni. 2013. Determination of crop evapotranspiration of table grapes in semi-arid of Northwest Mexico using multispectral vegetation index. Agric. Water Manage. 122: 12-19.

Ezzahar, J., A. Chehbouni, S. Er-Raki, and L. Hanich. 2009. Combining a large aperture scintillometer and estimates of available energy to derive evapotranspiration over several agricultural fields in semi-arid regions. Plant Biosys. 143: 209-221.

Gebler, S., F. H. J. Hendricks, T. Pütz, H. Post, M. Schmidt, and H. Vereecken. 2015. Actual evapotranspiration and precipitation measured by lysimeters: A comparison with eddy covariance and tipping bucket. Hydrol. Earth Syst. Sci. 19: 2145-2161.

Imukova, K., J. Ingwersen, M. Hevart, and T. Streck. 2016. Energy balance closure on a winter wheat stand: Comparing the eddy covariance technique with the soil water balance method. Biogeosciences 13: 63-75.

Liu, S. M., Z. W. Xu, Z. L. Zhu, Z. Z. Jia, and M. J. Zhu. 2013. Measurements of evapotranspiration from eddy-covariance systems and large aperture scintillometers in the Hai River Basin, China. J. Hydrol. 487: 24-38.

Liu, Y. and Y. Luo. 2010. A consolidated evaluation of the FAO-56 dual crop coefficient approach using the lysimeter data in the North China Plan. Agric. Water Manage. 97: 31-40.

López-Urrea, R., L. Martínez-Molina, F. de la Cruz, A. Montoro, J. González-Piqueras, M. Odi-Lara, and J. M. Sánchez. 2016. Evapotranspiration and crop coefficients of irrigated biomass sorghum for energy production. Irrig. Sci. 34: 287-296.

López-Avendaño, J. E., T. Díaz-Valdés, Ch. Watts-Thorp, J. C. Rodríguez, A. E. Castellanos-Villegas, L. Partida-Ruvalcaba y T. J. Velázquez-Alcaraz. 2015. Evapotranspiración y coeficientes de cultivo de chile bell en el Valle de Culiacán, México. Terra Latinoamericana 33: 209-219. 
Marine-Cirino, G., F. Justino, R. de Ávila-Rodríguez, and C. L. Teixeira-Andrade. 2015. Sensivity of the sorghum yield to individual changes in climate parameters: modelling based approach. Bragantia 74: 341-349. doi: 10.1590/4499.0411.

Martínez-Cob, A. and J. M. Faci. 2010. Evapotranspiration of a hedge-pruned olive orchard in a semiarid area of NE Spain. Agric. Water Manage. 97: 410-418.

Pereira, L. S., R. G. Allen, M. Smith, and D. Raes. 2015. Crop evapotranspiration estimation with FAO56: Past and future. Agric. Water Manage. 147: 4-20.

Piccinni, G., J. Ko, T. Marek, and T. Howell. 2009. Determination of growth-stage-specific crop coefficients $(\mathrm{Kc})$ of maize and sorghum. Agric. Water Manage. 96: 1698-1704.

Rosa, R. D., P. Paredes, G. C. Rodriguez, R. M. Fernando, I. Alves, L. S. Pereira, and R. G. Allen. 2012. Implementing the dual crop coefficient approach in interactive software: 2. Model testing. Agric. Water Manage. 103: 62-77.

Sadras, V. O. and D. Rodríguez. 2010. Modelling the nitrogendriven trade-off between nitrogen utilization efficiency and water use efficiency of wheat in Eastern Australia. Field Crops Res. 118: 297-305.

Samain, B., G. W. H. Simons, M. P. Voogt, W. Defloor, N. J. Bink, and V. R. N. Pauwels. 2012. Consistency between hydrological model, large aperture scintillometer and remote sensing based evapotranspiration estimates for heterogeneous catchment. Hydrol. Earth Syst. Sci. 16: 2095-2107.

Sánchez, J. M., R. López-Urrea, E. Rubio, and V. Caselles. 2011. Determining water use of sorghum from two-source energy balance and radiometric temperatures. Hydrol. Earth Syst. Sci 15: 3061-3070. doi: 10.5194/hess-15-3061-2011.

SEMARNAT-CONAGUA (Secretaría de Medio Ambiente y Recursos Naturales-Comisión Nacional del Agua). 2017. Estadísticas agrícolas de los Distritos de riego. Año agrícola 2015-2016. https://files.conagua.gob.mx/conagua/ publicaciones/Publicaciones/EA_2015-2016.pdf. (Consulta: enero 28, 2019).
Shenkut, A., K. Tesfaye, F. Abegaz, and T. Hordofa. 2013. Determination of water requirement and crop coefficient for sorghum (Sorghum bicolor L.) at Melkassa, Ethiopia. East Afr. J. Sci. 7: 41-50.

SIAP (Sistema de Información Agroalimentaria y Pesquera). 2016. Producción agrícola. http://www.gob.mx/siap/acciones-yprogramas/produccion-agricola-33119?idiom=es. (Consulta: noviembre13, 2016).

USDA (United States Department of Agriculture). 2016. Grain: World markets and Trade. http://apps.fas.usda.gov/psdonline/ circulars/grain.pdf. (Consulta: noviembre 3, 2016).

Williams, D. G., W. Cable, K. Hultine, J. C. B. Hoedjes, E. A. Yepez, V. Simonneaux, S. Er-Raki, G. Boulet, H. A. R. De Bruin, A. Chehbouni, O. K. Hartogensis, and F. Timouk. 2004. Evapotranspiration components determined by stable isotope, sap flow and eddy covariance tecniques. Agric. For. Meteorol. 125: 241-258.

Yimam, Y. T., T. E. Ochsner, and V. G. Kakani. 2015. Evapotranspiration partitioning and water use efficiency of switchgrass and biomass sorghum managed for biofuel. Agric. Water Manage. 155: 40-47.

Zhang, B., Y. Liu, D. Xu, N. Zhao, B. Lei, R. D. Rosa, P. Paredes, T. A. Paço, and L. S. Pereira. 2013. The dual crop coefficient approach to estimate and partitioning evapotranspiration of the winter wheat-summer maize crop sequence in North China Plain. Irrig. Sci. 31: 1303-1316. doi: 10.1007/s00271-0130405-1.

Zhao, P., S. Li, F. Li, T. Du, L. Tong, and S. Kang. 2015. Comparison of dual crop coefficient method and ShuttleworthWallace model in evapotranspiration partitioning in a vineyard of northwest China. Agric. Water Manage. 160: 41-56. 